\title{
Software como um Serviço: uma visão holística
}

\author{
Virgínia Maria Araújoํㅗㄹ Manuel Pérez Cota ${ }^{2}$ \\ varaujo@uatlantica.pt, mpcota@uvigo.es \\ ${ }^{1}$ Universidade Atlântica, Portugal, Fabrica da Pólvora Barcarena, 2730-036, Oeiras, Portugal \\ ${ }^{2}$ Universidade de Vigo, Campus Universitário, s/n, 36310 Vigo, Pontevedra, Espanha,
}

DOI: 10.17013/risti.19.145-157

\begin{abstract}
Resumo: A evolução tecnológica permite-nos dispor de soluções altamente flexíveis, de fácil acesso com valores de investimento mais baixos, o que leva a muitas empresas adotarem soluções SaaS (Software como um Serviço) para suporte aos seus processos de negócio. Hoje em dia, já quase todos os decisores de estratégias de TI nas organizações são levados a considerar a adoção de SaaS para suporte aos seus processos de negócio como uma solução de TI, pelos benefícios de conveniência associados à tecnologia ou competição no mercado. Muitas vezes, os decisores das estratégias de negócio consideram apenas os incentivos atraentes do uso de SaaS ignorando os impactos associados às novas tecnologias. É necessário ter uma visão holística das implicações no desenvolvimento, no fornecimento, na utilização e operacionalização de SaaS nos diferentes tipos de empresas. A presente pesquisa adota vários destes fatores de risco, como fatores dignos de avaliação de impacto do SaaS para comprovar a sua legitimidade com um estudo combinado quantitativo-qualitativo.
\end{abstract}

Palavras-chave: SaaS, Software como um Serviço, Paradigma, Interpretativo, Metodologia, Entrevista.

\section{Qualitative research to evaluate Software as a Service}

\begin{abstract}
Technological progress allows us to have highly flexible solutions, easily accessible with lower investment values, which leads many companies to adopt SaaS (Software as a Service) to support its business processes. Nowadays, almost all IT strategies decision makers in organizations are encouraged to consider the adoption of SaaS (Software as a Service) to support its business processes as an IT solution for the convenience benefits associated to the technology or market competition. Often the business strategies decision makers only consider the attractive incentives of SaaS and ignoring the impacts associated with new technologies. Is necessary to have a holistic view of the implications in the software development, supply, use and operation of SaaS in different types of business enterprises. This research adopts several of these risk factors, as worthy factors of SaaS impact assessment to prove its legitimacy with a quantitative and qualitative combined study.
\end{abstract}

Keywords: SaaS, Software as a Service, Paradigm, Interpretive, Methodology, Interview. 


\section{Introdução}

Nos inícios no atual milénio é oficialmente introduzido o termo SaaS. Embora, até hoje, ainda não exista um consenso acerca do conceito de SaaS, pode ser definido como um modelo de distribuição de Software que consiste no fornecimento de uma peça de Software, aplicação ou serviço, a múltiplos clientes ou inquilinos através da Internet (multi-tenant). A SIIA (Software and Information Industry Association) no artigo "Software as a Service: Strategic Backgrounder", diz - "[...] In the Software as a service model, the application or service, is deployed from a centralized data center across a network - Internet, intranet, LAN, or VPN - providing access and use on a recurring fee basis. Users "rent", "subscribe to", "are assigned" or "are granted access to" the applications from the central provider" (SIIA, 2001). SaaS é uma alternativa bastante atrativa de negócio para as pequenas, médias e grandes empresas. A abordagem do SaaS tem o potencial de transformar a forma com que os departamentos de tecnologia da informação (TI) se relacionam entre si e até o que pensam sobre o seu papel como fornecedores de serviços (Chong et al. 2006; Sá et al., 2014). Existem, no entanto, ainda várias preocupações e desafios que os fabricantes e fornecedores de SaaS têm de ultrapassar. Há que identificar logo de início, quais são as questões técnicas a ter em atenção e tudo aquilo que muda relativamente à construção de Software tradicional. A maioria das organizações conhece bem o valor da informação e o quão importante é a segurança dos dados. O cliente vai pagar pela utilização de um serviço que é configurado ou customizado de acordo com as suas especificações. Este pode, por exemplo, exigir que os seus dados estejam separados dos restantes clientes. Neste caso, o fornecedor tem de garantir o total isolamento que pode passar pela separação dos dados em base de dados própria para aquele cliente. Outro dos aspetos a considerar no desenvolvimento deste tipo de Software é o modelo de negócios a utilizar que pode simplesmente envolver custos maiores ou questões de construção do Software a considerar.

\section{Início do estudo e identificação do problema}

O surgimento de novas tecnologias e de modernos componentes e aplicações podem reduzir drasticamente o tempo de mercado e o custo para a conversão de um produto do modelo tradicional On-Premise (instaladas no local) numa solução SaaS. Contudo, apesar de surgirem várias ofertas de SaaS, nas soluções de "Software como um serviço" continua a subsistir um problema de isolamento, configuração, segurança e escalabilidade dos inquilinos. De fato, a escalabilidade da solução para cada cliente é na maioria das vezes efetuada "ad hoc", o que dá origem a problemas de desempenho, isolamento e escalabilidade. Identificam-se várias questões para as quais não temos resposta. Para iniciar um estudo no sentido de encontrar estas respostas, torna-se necessário restringir o âmbito da amostra alvo do estudo. Assim, pela proximidade e facilidade de comunicação aliada a uma apetência para a inovação, decide-se restringir o estudo a uma zona geográfica - Portugal. Para responder às questões da pesquisa, pretendese estabelecer um envolvimento com os donos ou gestores de empresas portuguesas no sentido de avaliar os impactos do uso de SaaS nas suas organizações, confirmando em paralelo a hipótese de que o SaaS traz benefícios para as empresas portuguesas e potencia o seu desenvolvimento. Procuramos na amostra abordar os aspetos do SaaS sobre diferentes pontos de vista para assim ter uma visão mais ampla e mais realista da 
questão de fundo desta investigação. Pretende-se avaliar o nível de adoção do SaaS nas empresas, se conhecem, se estão a usar e de que forma, que vantagens encontram em soluções deste tipo, em que áreas estão a utilizá-lo, que expectativas têm em termos de ganhos com SaaS. Pretende-se efetuar uma análise detalhada com vista a identificar os fatores necessários para o desenvolvimento da indústria de SaaS em Portugal e como o SaaS contribui para o aumento da eficiência e eficácia das organizações. São também identificados casos de sucesso de Utilizadores de SaaS, de Fabricantes e Fornecedores de SaaS.

\section{Questões da pesquisa e hipótese}

A questão de fundo analisada neste estudo é a seguinte: "Qual é o impacto do SaaS no desenvolvimento e crescimento das empresas?” Desta grande questão, surgem as seguintes questões:

- QP 1: quais são as reais necessidades no mercado de Software?

- QP 2: quais os receios e o que se está a fazer para os mitigar?

- QP 3: quais as implicações da adoção do SaaS?

- QP 4: onde devemos focar a atenção na oferta de SaaS no sentido de criar maior valor?

\section{Objeto de estudo, motivação e importância da pesquisa}

Hoje e cada vez mais as empresas têm de ser mais eficientes, mais competitivas, sendo necessário identificar todos os recursos que apoiem o seu desenvolvimento. Para além das tecnologias de otimização de Data Centers corporativos, existe hoje uma razoável oferta de soluções de SaaS. Este estudo constitui uma importante fonte de informação para as empresas que pretendem abordar os mercados de SaaS, para os fabricantes e fornecedores de Software conhecerem quais os receios e preocupações das empresas e daí desenvolverem esforços, no sentido de colmatar eventuais falhas nas suas soluções. Estes esforços podem implicar maiores investimentos em segurança, em customização, em isolamento de dados, em modelos de subscrição e cobrança mais simples, algumas alterações organizacionais e de cultura da empresa, etc. Como resultado desta investigação, são identificados diferentes tipos de aspetos de negócio, operacionais, funcionais, não funcionais, e técnicos. São identificados fatores interdependentes e inter-relacionados que são necessários avaliar quando se fala de SaaS. É objetivo deste estudo, ter o envolvimento dos gestores, responsáveis e decisores das empresas, avaliar os impactos da adoção de soluções SaaS nas suas organizações e confirmar a hipótese de que o SaaS traz benefícios e potencia o seu desenvolvimento. Efetuar uma recolha de feedback, tanto daqueles que utilizam como daqueles que consomem SaaS mas, também daqueles que desenvolvem e/ou fornecem Software SaaS, abrangendo desta forma os dois pontos de vista no comércio do Software. Pretende-se perceber o que de facto constitui um problema e o que efetivamente as empresas gostariam de ter disponível nesta oferta, e quais as suas implicações. Este estudo usa uma estratégia de pesquisa com base na combinação de métodos de investigação de forma sequencial. Primeiramente, é feito um levantamento para obter uma visão mais ampla e para fornecer informações de base através de uma pesquisa quantitativa (Denzin et al, 2005) 
que permite, posteriormente, ter uma maior profundidade na pesquisa quantitativa, ajudando a interpretar e contextualizar os resultados qualitativos.

\section{Paradigma da Pesquisa, Desenho e Método}

Segundo o dicionário, paradigma em investigação é “um sistema ou modelo conceptual que orienta o desenvolvimento posterior das pesquisas, estando na base da evolução científica. Designa-se então "paradigma” como um sistema de crenças, de princípios e de valores. Constitui um referencial filosófico que informa a metodologia utilizada, definindo o modelo daquilo que é investigado e como é investigado num determinado contexto epistemológico (Denzin, Lincoln, Yvonna, 2005, pp. 118-137). Essas crenças influenciam a forma como o investigador observa, analisa e interpreta o mundo e tudo aquilo que com ele interage. $\mathrm{O}$ investigador molda assim a sua visão sobre as coisas com base num conjunto de premissas epistemológicas e ontológicas e metodológicas. O conjunto destas premissas epistemológicas, ontológicas e metodológicas pode ser denominado de paradigma, ou esquema interpretativo, um "conjunto básico de crenças que orientam a ação" (Guba, 1990, p. 17). Qualquer pesquisa é orientada por um conjunto de crenças, de valores e princípios que o investigador usa na sua relação com o mundo, orientando-o na forma como o analisa e estuda. Cada paradigma exige esforços específicos, incluindo as questões propostas, as análises e interpretações que são feitas. O paradigma positivista ou quantitativo faz uma suposição das leis gerais que regem os fenómenos investigados. Formula as hipóteses, usa técnicas de verificação sistemática, procura explicações causais para os fenómenos e produz generalizações teóricas com validade e confiabilidade, recusando a compreensão subjetiva dos fatos de uma realidade (Denzin, Lincoln, Yvonna, 2005). O paradigma pós-positivista, interpretativo ou qualitativo trabalha com crenças, valores, opiniões, representações, práticas, lógicas de ação, atitudes e normas culturais. O objetivo é conseguir um entendimento profundo com um certo grau de subjetividade dos sujeitos (individual ou coletivo) e dos fenómenos, orientando a pesquisa para grupos reduzidos, mas a seremintensamente estudados (Denzin, Lincoln, Yvonna, 2005). Segundo o trabalho de (Bakos, 1987) a investigação baseada num ideal positivista experimental tem sido a abordagem dominante para os estudos de tecnologia da informação. Apesar de não ser o paradigma dominante, os métodos qualitativos e perspetivas interpretativas têm sidobastante usados em investigação de sistemas de informação. Segundo Yin (1984), os métodos qualitativos são caracterizados pelos seguintes fatores:

1. Observação detalhada ou envolvimento no ambiente natural em que o estudo ocorre;

2. Tentativa de evitar o compromisso prévio de construções teóricas ou hipóteses formuladas antes de recolher quaisquer dados (Yin R. K., 1984).

Dependendo daquilo que se pretende investigar, ambas as abordagens qualitativa e quantitativa, podem complementar-se permitindo dessa forma atingir os objetivos da pesquisa de forma mais eficaz. As abordagens podem ser complementares e adequadas para minimizar a subjetividade e aproximar o pesquisador do objeto de estudo, respondendo às principais críticas da abordagem qualitativa e quantitativa respetivamente, proporcionando maior confiabilidade aos dados (Hayati et al., 2006). Cavaye e Myers defendem que uma abordagem que combina dados quantitativos e 
qualitativos é totalmente aceite em pesquisas no âmbito dos Sistemas de Informação (Cavaye, 1996; Myers, 1999; Myers, 2003). No que refere à investigação qualitativa, Palvia, Mao, Salam, e Soliman referem que o estudo de caso é um dos métodos mais significativos na investigação em Sistemas de Informação. Regista-se uma tendência de crescimento na utilização deste método de estudo nesta área nos últimos anos, o que nos leva a concluir que os investigadores de SI estão cada vez mais interessados em obter conhecimento científico através da análise de comportamento em ambientes do mundo real (Palvia, Mao, Salam, Soliman, 2003, pp. 289-309). O estudo de caso segue uma estratégia de pesquisa que se centra na compreensão de um fenómeno no seu ambiente natural. Yin diz que o estudo de caso baseia-se em múltiplas fontes de evidência e múltiplas técnicas de recolha de dados, que podem ser documentos, arquivos, entrevistas, observação direta, observação participante e artefactos físicos (Yin R. K., 2011, pp. 474-475). Deve-se ter em atenção a subjetividade na recolha de dados e no processo de análise, que é considerada uma das principais desvantagens deste método. Segundo (Yin R., 2003) um projeto de pesquisa envolve o processo de recolha, medição, análise de dados, as conclusões que são extraídas e as recomendações que são feitas com base nessas conclusões de forma a combinar a relevância das questões da pesquisa. Dependendo daquilo que se pretende investigar, ambas as abordagens qualitativa e quantitativa, podem complementar-se permitindo dessa forma ao investigador atingir os seus objetivos de forma mais eficaz. Em diferentes fases do estudo ou até na mesma fase, pode-se adotar diferentes epistemologias e estilos de pesquisa. As abordagens podem ser complementares e adequadas para minimizar a subjetividade e aproximar o pesquisador do objeto de estudo, respondendo às principais críticas da abordagem qualitativa e quantitativa respetivamente, proporcionando maior confiabilidade aos dados (Hayati, Karami, Slee, 2006, pp. 361-394). Cavaye e Myers defendem que uma abordagem que combina dados quantitativos e qualitativos é totalmente aceite em pesquisas no âmbito dos Sistemas de Informação (Cavaye, 1996), (Myers, 1999, pp. 1-20) (Myers, 2003). Através da combinação de métodos, complementando a desvantagem de um com as vantagens de outro, aumenta-se a credibilidade e relevância da pesquisa. Hayati, Karami, Slee (2006) defendem que uma abordagem combinada de métodos quantitativos com métodos qualitativos que se complementam, enriquecendo a investigação. Já em 1959, Campbell e Fiske usam vários métodos para estudar a validade de traços psicológicos, utilizando uma abordagem combinada destes métodos, encorajando outros a utilizar a sua "matriz multi-método" para a recolha de dados no âmbito de um estudo. Isso leva outros investigadores a misturarem métodos. Surgem então associações de métodos de campo, tais como observações e entrevistas (dados qualitativos) com métodos de opinião tradicionais, tais como inquéritos (dados quantitativos) (Creswell, 2009). Também Hayati, Karami e Slee referem que a combinação de métodos, quantitativos e qualitativos, contribui para uma maior confiabilidade aos dados, complementando-se e colmatando as limitações de um método como outro método (Hayati, Karami, Slee, 2006). Creswell refere que os investigadores sentem que os preconceitos inerentes a um método único podem neutralizar os preconceitos de outros métodos. A partir do conceito original de triangulação das diferentes fontes de dados, vão nascendo cruzamentos de métodos quantitativos com métodos qualitativos. Os métodos mistos são mais complexos e exigem procedimentos mais explícitos (Creswell, 2009, p. 208). A presente pesquisa usa uma estratégia sequencial como mostra a Fig.1. Primeiramente é 
feito um levantamento para obter uma visão mais ampla e para fornecer informações de base que permitem uma maior profundidade nas entrevistas.

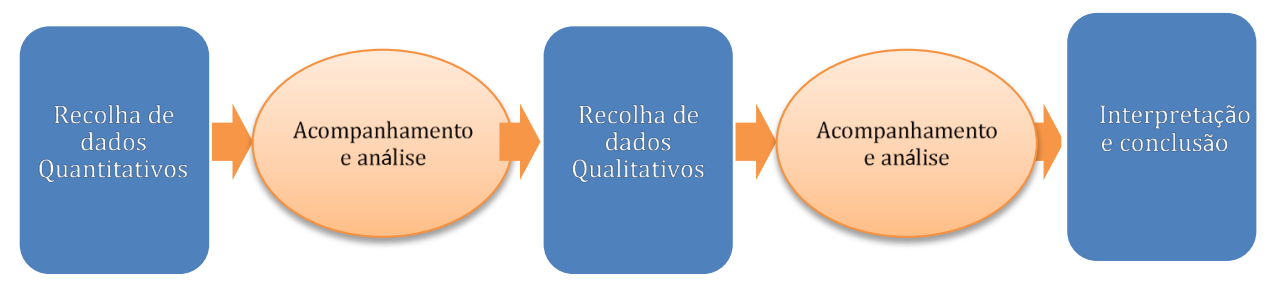

Figura 1 - Desenho sequencial dos métodos (adaptado de Creswell, 2009, p. 215)

Nos métodos sequenciais os dados quantitativos são analisados primeiro através de métodos estatísticos, e são usados para determinar as descobertas que se pretendem obter na fase seguinte (Driscol, Appiah-Yeboah, Salib, Rupert, 2007, pp. 19-28). Cresweel e Clark sugerem os seguintes passos para a implementação de uma pesquisa explanatória (Creswell, Clark, 2007). Para responder às questões da pesquisa e perceber se de facto as empresas portuguesas estão a tirar proveito do modelo de disponibilização do Software como um Serviço, em que medida melhora a sua eficiência e se é ou pode ser um fator determinante para a sua estratégia de crescimento conclui-se ser mais adequado adotar uma abordagem quantitativa, desenvolvendo um estudo profundo de uma recolha de dados alargada a várias empresas portuguesas. Para entender os diferentes tipos de empresas portuguesas segundo a perspetiva do decisor, do gestor ou diretor, dentro do seu contexto operacional particular, na sua realidade, chega-se à conclusão que é adequado complementar o estudo com uma abordagem qualitativa, aumentando desta forma a relevância da pesquisa. Este estudo usa uma estratégia de pesquisa com base na combinação de métodos de investigação de forma sequencial.

\subsection{O estudo de Casos}

A pesquisa qualitativa envolve o uso de dados qualitativos, recolhidos através de técnicas como entrevistas, documentos e dados de observação participante, de compreender e explicar fenómenos no seu contexto real. O método de Estudo de Caso adequa-se muito bem à pesquisa dos Sistemas de Informação nas organizações. Há uma preocupação organizacional para além das questões técnicas (Myers, 1997, pp. 241-242). Dentro do domínio dos Sistemas de Informação, têm surgido mais pesquisas a questões de foro organizacional e de gestão, distanciando-se das questões tecnológicas e daí o crescente interesse na aplicação de métodos de pesquisa qualitativa (Myers, 2003). São efetuados quatro estudos de caso, contextualizando desta forma a investigação no seio de cada organização e no seu ambiente real. Nesta abordagem qualitativa, recorre-se à técnica de entrevista a quatro casos selecionados através de amostragem intencional de uma lista de empresas identificadas através de redes sociais, boca-a-boca e referências profissionais. Os dados são recolhidos através de entrevista semiestruturada, face-a-face com os diretores/gestores responsáveis pela estratégia de TI da sua organização ou área de negócio. São selecionados os seguintes tipos de empresa: 


\begin{tabular}{llllll}
\hline Caso & Área de Negócio & Caracterização & $\begin{array}{l}\text { Ano } \\
\text { Criação }\end{array}$ & Empregados & Âmbito \\
\hline 1 & $\begin{array}{l}\text { Desenvolvimento } \\
\text { Software }\end{array}$ & $\begin{array}{l}\text { Integrada num } \\
\text { grande grupo } \\
\text { empresarial }\end{array}$ & 2000 & $>40.000$ & Nacional \\
\hline 2 & $\begin{array}{l}\text { Desenvolvimento } \\
\text { Software }\end{array}$ & PME Privada & 2011 & 10 & Nacional \\
\hline 3 & $\begin{array}{l}\text { Retalho Alimentar e } \\
\text { não Alimentar }\end{array}$ & $\begin{array}{l}\text { Grande Empresa } \\
\text { Privada }\end{array}$ & 1959 & $>40.000$ & Internacional \\
\hline 4 & $\begin{array}{l}\text { Desenvolvimento } \\
\text { Software }\end{array}$ & PME Privada & 2004 & 60 & Nacional \\
\hline
\end{tabular}

Tabela 1 - Perfil dos Casos selecionados

\subsection{Recolha, análise e interpretação dos dados}

Como instrumentos da pesquisa quantitativa, são desenvolvidos dois inquéritos Online distribuídos a uma amostra significativa de empresas portuguesas utilizadores, fabricantes e/ou fornecedores de "Software como um serviço". Todos os itens de ambos os questionários são pré testados, no sentido de testar os inquéritos e fazer uma revisão direcionando os aspetos da investigação. No estudo de caso, recorre-se à técnica de entrevista. A entrevista é um instrumento Standard de recolha de dados e uma fonte primária de informação em pesquisas de estudos de caso (Yin R., 2003). É garantida a todos os participantes a confidencialidade das informações prestadas e acordado que só é publicada a informação que o participante autoriza. Todos os participantes são informados dos objetivos da investigação, do propósito da entrevista e da sua participação, tendo inclusivamente assinado um termo de consentimento informado. Cada entrevista é áudio-gravada e dura cerca de uma hora e meia. Durante a entrevista qualitativa são tomadas notas das observações feitas pelo entrevistador / pesquisador. São realizadas entrevistas face-a-face para estabelecer rapport, construir confiança e para identificar quaisquer sinais não-verbais que justifiquem mais interrogatório. Os dados quantitativos são analisados através de ferramentas estatísticas que possibilitam a sua análise. A análise dos dados qualitativos começa logo no decorrer da entrevista. As notas de entrevista e as respostas às perguntas abertas são analisadas pelo método comparativo (Glaser, Strauss, 1967). É efetuado o cruzamento da informação recolhida nos inquéritos e nas entrevistas. É usado como base um quadro descritivo com os temas dos dados recolhidos. Procede-se posteriormente à análise do conteúdo da informação identificando a informação coerente e importante padronizando os dados recolhidos. É elaborada uma interpretação sumária de cada um dos casos. Segundo (Yin R. K., 1981, pp. 58-65) antes de iniciar a narrativa deve-se identificar os tópicos de organização da narrativa e de que forma a evidência deve ser integrada. Embora os estudos de caso possam muitas vezes começar com pequenos quadros conceptuais devem, contudo, ser organizados com base em proposições específicas, questões, considerações. Quanto às evidências, Yin diz também que os estudos de caso podem ser feitos usando qualquer evidência qualitativa ou quantitativa. Para a integração das evidências, os dados quantitativos e qualitativos que abordam o mesmo tema, 
devem ser referenciados juntos. Da mesma forma, devem-se integrar os segmentos de entrevistas com diferentes entrevistados, mas sobre o mesmo tema. Para evitar os problemas de análise de dados, estes devem ser codificados e tabulados. O trabalho produz estudos de caso de organizações que explicam o uso do Software como um serviço, nas diferentes vertentes de fabricantes, fornecedores e utilizadores. As questões dos inquéritos online e das entrevistas semiestruturadas são numeradas e agrupadas e estruturadas de acordo com o quadro descritivo, por forma a permitir a análise entre os casos analisados. A transcrição das entrevistas é enviada aos participantes tendo sido solicitada a confirmação da informação fornecida durante as entrevistas, para eliminar qualquer mal-entendido por parte do investigador. As entrevistas semiestruturadas seguem os mesmos tópicos em cada um dos casos. Os participantes são autorizados a falar sem interrupções e são interrompidos apenas para esclarecimento das questões que se proporcionam colocar. $\mathrm{O}$ investigador mantém uma atitude de neutralidade e rapport com cada um dos entrevistados. Rapport é uma posição frente a frente da pessoa a quem está a ser realizada a entrevista. Neutralidade é uma posição frente a frente face ao conteúdo daquilo que a pessoa diz (Patton, 2002, pp. 365-366). A análise dos dados embora já tenha começado durante a execução das entrevistas dá o seu arranque na fase de codificação da informação recolhida. Segundo (Patton, 2002, pp. 365-366), devese reduzir o volume de informação em bruto, identificar os padrões significativos, para construir uma estrutura de comunicação da essência do que os dados revelam. A melhor forma de avaliar resultados é identificar os diferentes padrões nos dados da pesquisa. Nesta fase são efetuadas as comparações entre os casos e o cruzamento da informação, no sentido de identificar aquilo que é comum a todos os casos e aquilo que é específico de um caso em particular, dentro das categorias identificadas no quadro descritivo do Framework de avaliação. Através desta análise dos dados, são identificados os padrões e os seus desvios. O resultado desta fase são as conclusões objetivas retiradas da análise dos dados recolhidos.

\section{5•3. Apresentação de Resultados}

No presente estudo começa-se por caracterizar a amostra do estudo, quer para a análise quantitativa (empresas Fabricantes, Fornecedoras, Utilizadoras de SaaS) quer para a análise qualitativa (Tab.1), para aborda-las no sentido de questiona-las quanto a este novo paradigma tecnológico. Os dados quantitativos, cujo detalhe está fora do âmbito deste artigo, são recolhidos através de inquéritos Online ao longo de cerca de 1 ano e têm cerca de 50 questões que abordam os temas em análise (Cota et al, 2014). Desta análise quantitativa conclui-se fundamentalmente que há um desconforto relativo à segurança dos dados, customização e integração; Há ainda uma grande renitência em migrar processos críticos de negócio para SaaS, embora muitas empresas já usem SaaS para processos importantes; Os principais motivos que levam ou que podem levar as empresas a utilizar Software SaaS são a poupança nos custos Hardware, Software e suporte de TI; Do ponto de vista dos Fabricantes/fornecedores de SaaS, consideram a oferta SaaS necessária para garantir o seu posicionamento no mercado de Software; Existem várias preocupações no que refere ao nível do serviço prestado pelos fornecedores do Software; Muitos utilizadores desconhecem o conceito e o valor do SaaS; Os principais desafios no desenvolvimento e fornecimento de soluções de SaaS são a privacidade dos dados dos clientes, a integração com outras aplicações, a customização da aplicação para cada 
cliente e a conetividade e acesso; A maioria dos Fornecedores de SaaS não têm modelos arquiteturais maduros que lhes permita aumentar o benefício reduzindo o custo. Da análise qualitativa realizada na sequeência, resulta um quadro comparativo dos casos estudados em termos de requisitos não funcionais (Tab.2 e 3) e requisitos técnicos (Tab. 4 e 5). Para perceber o que de facto constitui um problema no SaaS e o que efetivamente as empresas gostariam de ter disponível nesta oferta. Os resultados revelam que o SaaS efetivamente constitui uma solução bastante interessante e sólida no desenvolvimento das empresas, contudo, existe a necessidade de maiores esforços nomeadamente ao nível da possibilidade de customização para cada cliente (Tenant) e a integração da aplicação SaaS com as aplicações On-Premise (instaladas no local).

\begin{tabular}{|c|c|c|c|c|}
\hline Empresa & $\begin{array}{l}\text { Qualidade } \\
\text { do serviço }\end{array}$ & $\begin{array}{l}\text { Benefícios e } \\
\text { eficiência }\end{array}$ & Confiabilidade & Redução de custos \\
\hline Caso1 & Nível elevado & Produtividade & $\begin{array}{l}\text { Redundância de } \\
\text { infraestrutura, } \\
\text { aplicações e dados. } \\
\text { Sem interrupção. }\end{array}$ & $\begin{array}{l}\text { Custo de setup mais baixo e } \\
\text { às vezes zero, com pagamento } \\
\text { mensal, anual, ou de utilização. }\end{array}$ \\
\hline Caso 2 & $\begin{array}{l}\text { Apresenta } \\
\text { qualidade }\end{array}$ & Colaboração & $\begin{array}{l}\text { Redundância de } \\
\text { infraestrutura, } \\
\text { aplicações e dados. }\end{array}$ & $\begin{array}{l}\text { Cada contabilista por pertencer } \\
\text { à Ordem tem direito a } 3 \text { licenças } \\
\text { de subscrição. }\end{array}$ \\
\hline Caso 3 & $\begin{array}{l}\text { Apresenta } \\
\text { qualidade }\end{array}$ & $\begin{array}{l}\text { Melhoria de } \\
\text { processo }\end{array}$ & $\begin{array}{l}\text { Fornecedor credível } \\
\text { com elevado n } \\
\text { de clientes a nível } \\
\text { mundial, }\end{array}$ & $\begin{array}{l}\text { Custos indiretos. Redução da } \\
\text { formação em sala e aumento da } \\
\text { formação E-learning. }\end{array}$ \\
\hline Caso 4 & $\begin{array}{l}\text { Apresenta } \\
\text { qualidade }\end{array}$ & $\begin{array}{l}\text { Acompanhamento } \\
\text { do TtM }\end{array}$ & $\begin{array}{l}\text { Credibilidade } \\
\text { dos parceiros } \\
\text { fabricantes de SaaS }\end{array}$ & $\begin{array}{l}\text { Poupanças em custos de } \\
\text { infraestrutura próprios }\end{array}$ \\
\hline
\end{tabular}

Tabela 2 - Requisitos não funcionais

\begin{tabular}{|c|c|c|c|c|}
\hline Empresa & Modelo de pagamento & $\begin{array}{l}\text { Tempo de } \\
\text { mercado }\end{array}$ & Implicações & Satisfação \\
\hline Caso1 & $\begin{array}{l}\text { Horas homem; Subscrição; } \\
\text { Utilização } \\
\text { Medição de utilização. }\end{array}$ & $\begin{array}{l}\text { SaaS é } \\
\text { competência core } \\
\text { da empresa }\end{array}$ & $\begin{array}{l}\text { Dificuldades na } \\
\text { aceitação deste tipo } \\
\text { de oferta no mercado } \\
\text { português }\end{array}$ & Sim \\
\hline Caso 2 & $\begin{array}{l}\text { Mensal; } \\
\text { Subscrição }\end{array}$ & $\begin{array}{l}\text { SaaS é } \\
\text { competência core } \\
\text { da empresa }\end{array}$ & $\begin{array}{l}\text { Dificuldades na } \\
\text { distribuição do } \\
\text { Software e com } \\
\text { pessoal capacitado. }\end{array}$ & Sim \\
\hline Caso 3 & Subscrição & $\begin{array}{l}\text { Facilidade em } \\
\text { novos processos } \\
\text { de gestão. }\end{array}$ & $\begin{array}{l}\text { Conteúdos } \\
\text { E-learning mais } \\
\text { caros. }\end{array}$ & Sim \\
\hline Caso 4 & Subscrição & $\begin{array}{l}\text { Evolução } \\
\text { tecnológica; } \\
\text { Gestão mais } \\
\text { simples e TtM }\end{array}$ & $\begin{array}{l}\text { Novo modelo de } \\
\text { projetos; } \\
\text { Alterações na } \\
\text { prestação de serviço e } \\
\text { cultura, }\end{array}$ & $\begin{array}{l}\text { Sim mas pode } \\
\text { melhorar }\end{array}$ \\
\hline
\end{tabular}

Tabela 3 - Requisitos não funcionais (continuação) 


\begin{tabular}{llllll}
\hline Empresa & $\begin{array}{l}\text { Níveis } \\
\text { funcionalidades, } \\
\text { atualizações } \\
\text { roadmap }\end{array}$ & Customização & Integração & Arquitetura & Segurança \\
Caso 1 & $\begin{array}{l}\text { Releases mensais e } \\
\text { trimestrais }\end{array}$ & Pouca & Sim & $\begin{array}{l}\text { Multi-Tenant } \\
\text { aplicacional e } \\
\text { dados }\end{array}$ & $\begin{array}{l}\text { Encriptação } \\
\text { Autenticação }\end{array}$ \\
\hline Caso 2 & $\begin{array}{l}\text { Atualizações } \\
\text { frequentes }\end{array}$ & Não & Em parte & $\begin{array}{l}\text { Multi-Tenant } \\
\text { Aplic. } \\
\text { single-tenant } \\
\text { dados }\end{array}$ & $\begin{array}{l}\text { Encriptação } \\
\text { Autenticação }\end{array}$ \\
\hline Caso 3 & 3 Releases anuais & Não & Sim & Encriptação \\
Caso 4 & Releases anuais & Pouca & Pouca & $\begin{array}{l}\text { Multi-Tenant } \\
\text { aplicacional e } \\
\text { dados }\end{array}$ & $\begin{array}{l}\text { Encriptação } \\
\text { Autenticação }\end{array}$ \\
\hline
\end{tabular}

Tabela 4 - Requisitos técnicos

\begin{tabular}{lllll}
\hline Empresa & Disponibilidade & Virtualização & Tecnologias & $\begin{array}{l}\text { Tendência emergente na } \\
\text { indústria de Software }\end{array}$ \\
\hline Caso 1 & $\begin{array}{l}\text { Disaster Recovery } \\
\text { Sem interrupção }\end{array}$ & Sim, Vmware & $\begin{array}{l}\text { Java, Net, } \\
\text { Webservices, SOA, } \\
\text { Mobile }\end{array}$ & Globalização \\
\hline Caso 2 & $\begin{array}{l}\text { Disaster Recovery } \\
\text { Sem interrupção }\end{array}$ & Sim, Vmware & $\begin{array}{l}\text { Open Source, Linux, } \\
\text { Postgres, C, Java, } \\
\text { Ruby, HTML5 }\end{array}$ & Mobilidade \\
\hline Caso 3 & $\begin{array}{l}\text { Disaster Recovery } \\
\text { Sem interrupção }\end{array}$ & & Cornerstone & $\begin{array}{l}\text { Oferta modular, integrada; } \\
\text { Customização }\end{array}$ \\
\hline Caso 4 & $\begin{array}{l}\text { Disaster Recovery } \\
\text { Sem interrupção }\end{array}$ & $\operatorname{Sim}$ & $\begin{array}{l}\text { Dynamics NAV, } \\
\text { CRM, Business } \\
\text { Objects; Sana Com }\end{array}$ & $\begin{array}{l}\text { Mobilidade; tandartizaçao } \\
\text { arquitetura SaaS }\end{array}$ \\
\hline
\end{tabular}

Tabela 5 - Requisitos técnicos (Continuação)

\section{Conclusões e contribuições}

Articulando os dois estudos efetuados (quantitativo e qualitativo), concluímos que de facto os clientes de SaaS devem definir as suas próprias estratégias de integração de SaaS e devem incorporá-las numa aproximação holística à integração multiempresa, em todos os projetos (Lheureux, 2008). A identificação das maiores preocupações nas diferentes dimensões: técnica, negócio, organizacional e operacional, respondem às duas primeiras questões da pesquisa. A terceira questão, sobre as implicações da adoção do SaaS, é respondida com a análise efetuada e interpretação dos dados recolhidos. O estudo confirma a afirmação de Lheureux (2008) quanto à integração de SaaS nas estratégias de negócios. Confirma-se a afirmação de F. Chong, G. Carraro e R. Wolter, quanto ao facto da integração e a customização serem componentes críticas nas estratégias de uma arquitetura SaaS bem-sucedida, numa infraestrutura de TI centralizada em serviço 
(Chong et al, 2006), no entanto, existem muitas limitações de customização na oferta de SaaS analisada. Verifica-se a adoção de SaaS por médias e grandes empresas e não apenas pequenas empresas em contraste com estudos anteriores (Kern et al, 2002). Os resultados mostram que os gestores das empresas reconhecem o valor acrescentado e a mais-valia do "Software como um Serviço" nas suas organizações apesar de existirem necessidades de alteração, adaptação e evolução que podem ter implicações sobre a lógica de negócio do Fornecedor do Serviço. Confirma-se que de facto o SaaS pode transformar, positivamente, a forma de construção e disponibilização do Software. SaaS não é só um modelo diferente de distribuição de Software, é uma nova forma de fazer negócio com Software. A escalabilidade das soluções é central para atingir volumes críticos de forma a gerar os lucros pretendidos. O reconhecimento por parte do utilizador de que o SaaS funciona para a sua organização, para as suas necessidades, inicia um ciclo virtuoso de referências que se reflete num marketing viral. A segurança e privacidade dos dados são uma área de preocupação e insegurança quanto à utilização de SaaS. SaaS está a avançar rapidamente, o Time to Market(TtM) é mais importante do que nas soluções On-Premise. Os SLA dos serviços SaaS são muito importantes, os utilizadores ficam preocupados com os níveis de um serviço sobre o qual não têm controlo. Assim, ter uma visão transparente do serviço e do seu desempenho é muito importante, devem portanto ser disponibilizados relatórios de desempenho do serviço. Os modelos de licenciamento complexos podem ser um handicap (desvantagem). A realização de um estudo exaustivo, aprofundado e pormenorizado combinando as análises quantitativa e qualitativa, permite identificar um conjunto de conclusões sobre a temática em investigação segmentando a amostra dirigindo a pesquisa cuidada nas várias vertentes desde o desenvolvimento do Software, à sua distribuição e utilização. Mais ainda, é analisado um conjunto de questões de investigação no terreno, na realidade das empresas portuguesas. Através da combinação das análises efetuadas, que se complementam, temos desta forma, um estudo mais rico que traz novas descobertas e dá um forte contributo a vários níveis ao teatro do "Software como um Serviço”. Através da análise e interpretação dos resultados do estudo, juntamente com a análise prévia da literatura, são identificadas implicações que podem melhorar a prática empreendedora entre empresas portuguesas aumentando a sua a competitividade e desenvolvimento, e fornece também uma base para pesquisas futuras.

\section{Agradecimentos}

Quero expressar o meu agradecimento ao Prof. Dr. Manuel Pérez Cota, Prof. Dr. José Ayude Vázquez, Prof. Dr. Jacinto Dacosta, ao Dr. Miguel Zegre, ao Eng. ${ }^{\circ}$. Vítor Pinho, ao Dr. João Brás, ao Sr. Fernando Trigo, ao Eng. ${ }^{\circ}$. Rui Cardoso e ao Dr.Jorge Xavier.

\section{Referências}

Araujo, M. Virginia, Cota, M. Perez, Avaliando SaaS através de métodos combinados de investigação. Atas CIAIQ2016. Investigação Qualitativa em Engenharia e Tecnologia, Volume 4

Bakos, J. (1987). Dependent Variables for the Study of Firm and Industry-Level impacts of Information Technology. Proceedings of the Eighth International Conference on Information Systems. Pittsburgh, PA. 
Cavaye, A. (1996). Case Study Research: A Multi-Faceted Research Approach For IS. Information Systems Journal, 6(3), 227-242.

Bakos, J. (1987). Dependent Variables for the Study of Firm and Industry-Level impacts of Information Technology. Proceedings of the Eighth International Conference on Information Systems. Pittsburgh, PA.

Creswell, J. W., \& Clark, V. L. (2007). Designing and Conducting Mixed Methods Research. Thousand Oaks, California: Sage Publications Inc.

Creswell, J. W. (2009). Research design: Qualitative, Quantitative, and Mixed Methods Approaches. Tousand Oaks, california: Sage Publications Inc.

Chong, F., Carraro, G., \& Wolter, R. (2006). Software as a Service (SaaS): An Enterprise Perspective. Obtido em 03 de 2012, de MSDN Library: Microsoft Corporation: http://msdn.microsoft.com/en-us/library/aa905332.aspx

Chong, F., Carraro, G., \& Wolter, R. (2006). Software as a Service (SaaS): An Enterprise Perspective. Obtido em 03 de 2012, de MSDN Library: Microsoft Corporation: http://msdn.microsoft.com/en-us/library/aa905332.aspx

Costa, António Pedro et al. (2015). Envolvimento dos Utilizadores na definição de Requisitos: o caso da plataforma WEBMAT. RISTI - Revista Ibérica de Sistemas e Tecnologias de Informação, (E4), 92-106. Cota, M. Pérez, Thomaschewski, J., Schrepp, M., Gonzçalves, R. (2014). Efficient Measurement of the User Experience. A Portuguese Version. Procedia Computer Science 27, 491-498, 2014

Denzin, N., Lincoln , K., \& Yvonna, S. (2005). Handbook of qualitative research. Thousand Oaks CA, US: Sage Publications Inc.

Driscol, D. L., Appiah-Yeboah, A., Salib, P., \& Rupert, D. J. (2007). Merging Qualitative and Quantitative Data in Mixed Methods Research: How To and Why Not. Ecological and Environment Anthropology, 3(1), 19-28.

Glaser, B. G., \& Strauss, A. L. (1967). The Discovery of Grounded Theory: Strategies for Quaiitative Research. New york: Aldine.

Hayati, D., Karami, E., \& Slee, B. (2006). Combining qualitative and quantitative methods in the measurement of rural poverty. Social Indicators Research, 75(3), 361-394.

kern, T., Kreijger, J., \& Willcocks, L. (2002). Exploring ASP as sourcing strategy: theoretical perspectives, propositions for practice. Journal of Strategic Information Systems, 11(2), 153-177.

Lheureux, B. (2008). SaaS Integration: How to Choose the Best Approach. Gartner.

Myers, M. D. (1997). Qualitative Research in Information Systems (Vol. 21). MIS Quarterly.

Myers, M. D. (1999). Investigating Information Systems with Ethnographic Research. Communications of AIS, 2(23), 2-20.

Myers, M. D. (2003). Qualitative Research in Information Systems. MISQ Discovery. 
Palvia, P., Mao, E., Salam, A. F., \& Soliman, K. S. (2003). Management Information Systems Research: What's There In A Methodology. Communications Of The Association For Information Systems (CAIS), 11(16).

Patton, M. Q. (2002). Qualitative research \& evaluation methods (3rd ed.). Thousand Oaks, California: Sapge Publications Inc.

Sá, F., Rocha, Á., \& Cota, M. P. (2014, June). Quality models for online e-Government services. In 2014 9th Iberian Conference on Information Systems and Technologies (CISTI) (pp. 1-5). IEEE.

SIIA. (02 de 2001). Software as a Service: Software as a Service: . Obtido de siia.net: https://www.siia.net/estore/pubs/SSB-01.pdf

Souza, Dayse Neri de et al. Visão de Orientadores e Orientandos sobre o Software Online de Supervisão da Investigação - IARS. RISTI, Set 2015, no.spe4, p.66-78. ISSN 1646-9895

Yin, R. K. (1981). The Case Study Crisis: Some Answers (Vol. 26). Administrative Science Quarterly.

Yin, R. K. (1984). Case Study Research: Design and Methods (2nd ed.). Sage Publications Inc.

Yin, R. (2003). Case Study Research: Design And Methods (Vol. 5). Sage Publications Inc

Yin, R. K. (2011). Case Study Research: Design And Methods. The Modern language journal, 95(3). 